\title{
Phenotypic expression in von Hippel-Lindau disease: correlations with germline VHL gene mutations
}

Eamonn R Maher, Andrew R Webster, Frances M Richards, Jane S Green, Paul A Crossey, Stewart J Payne, Anthony T Moore

\begin{abstract}
Von Hippel-Lindau disease is an autosomal dominantly inherited familial cancer syndrome predisposing to retinal and central nervous system haemangioblastomas, renal cell carcinoma, and phaeochromocytoma. VHL disease shows variable expression and interfamilial differences in predisposition to phaeochromocytoma. In a previous study of 65 VHL kindreds with defined VHL mutations we detected significant differences between VHL families with and without phaeochromocytoma such that missense

for counselling VHL families and indicate that specific VHL mutations may be associated with different tumour susceptibility risks. There was no evidence of a generalised increase in age related tumour risks for missense mutations, suggesting that missense mutations predisposing to phaeochromocytoma have tissue specific effects, possibly because the VHL protein has several functions, the importance of which varies from tissue to tissue, or because the proteins which interact with VHL differ between different tissues.
\end{abstract} mutations were more common and large deletions or protein truncating mutations less frequent in phaeochromocytoma positive families. To investigate the significance and cause of this association further, we studied 138 VHL kindreds for germline mutations and calculated the age related tumour risks for different classes of VHL gene mutations. Using SSCP, heteroduplex and Southern analysis we identified a germline VHL gene mutation in 101 families (73\%). Direct sequencing of the VHL coding region further increased the mutation detection rate to $81 \%$. In addition to precise presymptomatic diagnosis, identification of a VHL gene mutation can provide an indication of the likely phenotype. We found that large deletions and mutations predicted to cause a truncated protein were associated with a lower risk of phaeochromocytoma (6\% and $9 \%$ at 30 and 50 years, respectively) than missense mutations $(40 \%$ and $59 \%$, respectively) and that missense mutations at codon 167 were associated with a high risk of phaeochromocytoma $(53 \%$ and $82 \%$ at ages 30 and 50 years). Cumulative probabilities of renal cell carcinoma did not differ between the two groups (deletion/ truncation mutations: $8 \%$ and $60 \%$, and missense mutations: $10 \%$ and $64 \%$ at ages 30 and 50 years, respectively). Age related risks for haemangioblastoma were similar in the two mutation groups, with the age related risks of cerebellar haemangioblastoma slightly less (35\% and $64 \% v 38 \%$ and $75 \%$ at ages 30 and 50 years) and retinal haemangioblastoma slightly higher (45\% and $72 \% v 37 \%$ and $64 \%$ at ages 30 and 50 years) in the missense mutation group than in the deletion/protein truncation group. These results provide valuable data
( $($ Med Genet 1996;33:328-332)

Key words: von Hippel-Lindau disease; VHL gene mutations; phaeochromocytoma; renal cell carcinoma.

Von Hippel-Lindau disease (VHL) is an autosomal dominant disorder with a birth incidence of 1 in 36000 per year. ${ }^{1}$ The major complications include haemangioblastomas of the central nervous system and retina, renal cell carcinoma, and phaeochromocytoma. ${ }^{23}$ In addition renal, pancreatic, and epididymal cysts are frequent. The expression of VHL disease is variable but clinical heterogeneity for phaeochromocytoma is well recognised with some families showing a high incidence of this tumour, while others have a very low frequency. The gene for VHL disease was mapped to the short arm of chromosome 3 in 1988 and was isolated by a positional cloning approach in $1993 .{ }^{45}$ Recently the VHL gene product has been shown to function as a negative regulator of transcriptional elongation. ${ }^{6-8}$ Following the isolation of the VHL gene and the identification of germline mutations in VHL families, we and others described a genotype-phenotype correlation such that there is a significantly greater incidence of missense mutations in patients with phaeochromocytoma. ${ }^{910}$ To investigate genotype-phenotype relationships in VHL disease further we studied a large series of patients to define the spectrum and optimum detection method of germline VHL mutations and analysed the cumulative risks of the major VHL associated tumours associated with different classes of VHL mutations.

Materials and methods

PATIENTS

A diagnosis of VHL disease was made using conventional criteria and all affected patients 
Table 1 Germline VHL gene mutations detected in this study

\begin{tabular}{|c|c|c|c|}
\hline Family ID & $D N A$ change & Protein change* & Phaeo \\
\hline 39 & $407 \mathrm{C} \rightarrow \mathrm{G}$ & Ser $65 \operatorname{Trp}$ & - \\
\hline 46 & 427 Ins GCCC & Frameshift & Not \\
\hline 112 & 427 Del T & Frameshift & No \\
\hline 38 & $430 \mathrm{C} \rightarrow \mathrm{T}$ & Gln $73 \mathrm{Ter}$ & No \\
\hline 74 & $430 \mathrm{C} \rightarrow \mathrm{T}$ & Gln 73 Ter & Yes \\
\hline 134 & 440 Del TCT & DelPhe 76 & - \\
\hline 144 & 440 Del TCT & DelPhe 76 & - \\
\hline 160 & $446 \mathrm{~A} \rightarrow \mathrm{G}$ & Asn 78 Ser & - \\
\hline 139 & $553+5 \mathrm{G} \rightarrow \mathrm{C}$ & Splicing defect & Noł \\
\hline 98 & $500 \mathrm{~A} \rightarrow \mathrm{C}$ & Giln 96 Pro & No \\
\hline 18 & $566 \mathrm{~T} \rightarrow \mathrm{G}$ & Leu 118 Arg & Yes $\$$ \\
\hline 127 & $545 \mathrm{G} \rightarrow \mathrm{A}$ & Ser 111 Asn & No \\
\hline 131 & $559 \mathrm{C} \rightarrow \mathrm{G}$ & Leu $116 \mathrm{Val}$ & - \\
\hline 97 & $564 \mathrm{G} \rightarrow \mathrm{A}$ & Trp 117 Ter & Nol \\
\hline 147 & 574 Del G & Frameshift & No \\
\hline 107 & $634 \mathrm{Del} A$ & Frameshift & No \\
\hline 96 & $694 \mathrm{C} \rightarrow \mathrm{T}$ & Arg 161 Ter & No \\
\hline 109 & $694 \mathrm{C} \rightarrow \mathrm{T}$ & Arg $161 \mathrm{Ter}$ & No \\
\hline 31 & $694 \mathrm{C} \rightarrow \mathrm{T}$ & Arg $161 \mathrm{Ter}$ & Nos \\
\hline 55 & $694 \mathrm{C} \rightarrow \mathrm{T}$ & Arg $161 \mathrm{Ter}$ & Nof̂ \\
\hline 42 & $698 \mathrm{G} \rightarrow \mathrm{A}$ & Cys $162 \mathrm{Tyr}$ & Noß̊ \\
\hline 50 & $709 \mathrm{G} \rightarrow \mathrm{T}$ & Val 166 Phe & Yes \\
\hline 153 & $712 \mathrm{C} \rightarrow \mathrm{T}$ & Arg 167 Trp & - \\
\hline 77 & $722 \mathrm{~T} \rightarrow \mathrm{A}$ & Val $170 \mathrm{Asp}$ & -6 \\
\hline 36 & $742 \mathrm{~A} \rightarrow \mathrm{T}$ & Arg $177 \mathrm{Ter}$ & Nos \\
\hline 37 & $742 \mathrm{~A} \rightarrow \mathrm{T}$ & Arg $177 \mathrm{Ter}$ & Yes§ \\
\hline 113 & 771-773 Del AGA & del Glu 186 & No \\
\hline 141 & 776 Del T & Frameshift & No \\
\hline
\end{tabular}

Phaeo=presence (Yes) or absence (No) of phaeochromocytoma in family.

* The codon numbers are taken from the revised numbering system and correspond to 71 less than the system used previously ${ }^{5910}$ (for example, codon 238 is now codon 167). $t=$ An insertion at nucleotide 331 was previously detected in this patient. ${ }^{13}$ $\ddagger=$ Predicted splicing defect but not confirmed by RNA analysis (not available) $\S=$ Mutation detected by DNA sequencing. had proven retinal angioma, central nervous system haemangioblastoma, renal cell carcinoma, or phaeochromocytoma. ${ }^{2}$

IDENTIFICATION OF VHL GENE MUTATIONS We have previously reported the identification of germline mutations in 77 of 116 unrelated VHL kindreds using Southern analysis to detect large deletions and single conformational polymorphism analysis (SSCP) and sequencing to detect small intragenic mutations. ${ }^{911}$ In this study a further 22 unrelated families were added to our mutation panel (total 138 kindreds) and molecular genetic analysis performed as described previously. In addition, DNA from patients in which a mutation had not been identified were investigated to determine if direct sequencing of the VHL gene could further improve the mutation detection rate.

\section{GENOTYPE-PHENOTYPE ANALYSIS}

The relationship between genotype and phenotype in 90 VHL kindreds with known germline mutations was analysed to determine if the previous association between phaeochromocytoma predisposition and missense mutations persisted in a larger sample. Furthermore, to investigate if this association was because missense mutations caused a more severe phenotype with earlier onset of phaeochromocytoma and other complications, we calculated cumulative risks for each of the major complications for patients with missense mutations $(n=100)$ and patients with germline deletions or mutations predicted to cause a truncated protein product $(n=157)$ using life table analysis methods. ${ }^{2}$
MOLECULAR GENETIC ANALYSIS

High molecular weight DNA was isolated from peripheral blood by conventional methods. Germline VHL gene mutations were identified by Southern analysis, SSCP, and heteroduplex analysis as described previously. ${ }^{911}$ In those families that were negative for VHL mutations by these methods the complete VHL coding region was sequenced using the Applied Biosystems $\mathrm{ABI}^{\mathrm{TM}}$ model 373 automated sequencer. The VHL coding region and $5^{\prime}$ untranslated region ${ }^{512}$ were amplified in three polymerase chain reactions for each of the three VHL exons, using primers reported previously, ${ }^{9}$ from leucocyte DNA. The amplified DNA was purified using Promega Wizard ${ }^{\mathrm{TM}}$ PCR purification columns and the double stranded DNA was used as the template for cycle sequencing. A total of $4.5 \mu \mathrm{l}$ of purified PCR product was added to $3.2 \mathrm{pmol}$ of oligonucleotide primer and $9.5 \mu$ l of Prism ${ }^{\mathrm{TM}}$ Ready Reaction Dye Deoxy ${ }^{\mathrm{TM}}$ Terminator Cycle Sequencing mix in a total volume of $20 \mu \mathrm{l}$. The reaction mixture was overlaid with mineral oil and cycle sequencing was performed on a Perkin Elmer Cetus Thermal Cycler for 35 cycles: $96^{\circ} \mathrm{C}$ for 30 seconds, $50^{\circ} \mathrm{C}$ for 30 seconds, and $60^{\circ} \mathrm{C}$ for four minutes. Dideoxy terminated DNA fragments were extracted using phenol: $\mathrm{H}_{2} \mathrm{O}$ :chloroform (68:18:14) and ethanol precipitated before analysis using a Model 373 $\mathrm{ABI}^{\mathrm{TM}}$ automated sequencer.

\section{Results}

IDENTIFICATION OF GERMLINE VHL GENE MUTATIONS

Southern, SSCP, and heteroduplex analysis of 138 unrelated VHL families showed germline mutations in 101 (73\%). Mutations in 77 kindreds have been reported previously, ${ }^{911}$ and details of the additional intragenic mutations are shown in table 1. Direct sequencing of the VHL coding region was performed in 28 probands in whom a germline mutation had not been identified by Southern, SSCP, and heteroduplex analysis. In eight patients a germline mutation was then identified (table 1). Each of these mutations resulted from a single nucleotide substitution, six of which had not been previously reported, but the $694 \mathrm{C} \rightarrow \mathrm{T}$ mutation had been detected by SSCP in some other families. The addition of VHL gene sequencing to Southern, SSCP, and heteroduplex analysis increased the VHL mutation detection rate to $81 \%$.

The 109 mutations comprised: 26 large deletions (19\% of all 138 patients), 37 missense mutations, 20 nonsense mutations, 18 frameshift insertions or deletions, six in frame deletions, and two splice site mutations. Of the 28 families and mutations in table 1,18 mutations in 20 families had not been found in our previous report and 15 of these mutations have not been described in other large series. ${ }^{1014}$ Among the 83 families with intragenic mutations there were 58 distinct mutations, the most common being C694T $(n=7)$, C712T $(n=6)$, G713A $(n=3)$, and delTCT440-442 $(\mathrm{n}=4)$. VHL mutations ${ }^{9}$ were non-randomly 
Table 2 Types of VHL gene mutations in phaeochromocytoma positive $(n=19)$ and negative ( $n=75)$ families. Missense mutations are significantly more frequent in the Phaeo + group $\left(\chi^{2}=15 \cdot 8, p<0 \cdot 0005\right)$ and deletions, nonsense and frameshift mutations are significantly more frequent in Phaeo-families $\left(\chi^{2}=10 \cdot 7, p<0 \cdot 005\right)$

\begin{tabular}{llc}
\hline Mutation & $\begin{array}{l}\text { Phaeo negative } \\
\text { No (\%) }\end{array}$ & $\begin{array}{l}\text { Phaeo positive } \\
\text { No (\%) }\end{array}$ \\
\hline Large deletion & $25(33)$ & $1(5)$ \\
Frameshift/nonsense & $29(39)$ & $5(26)$ \\
Missense & $16(21)$ & $13(68)$ \\
In frame deletion & $4(5)$ & $0(0)$ \\
Splice & $1(1)$ & $0(0)$ \\
\hline
\end{tabular}

distributed and no mutations were identified $5^{\prime}$ to nucleotide 375 in the published VHL sequence. ${ }^{5}$ Although we had previously reported a small insertion at nucleotide 331 in family $46,{ }^{9}$ we have subsequently identified a further frameshift insertion (GCCC) at nucleotide 427 in this family (table 1 ).

\section{GENOTYPE-PHENOTYPE CORRELATIONS}

Details on the presence or absence of phaeochromocytoma were available for 94 of the 109 families with identified VHL mutations. A comparison of germline mutations in families containing a person with phaeochromocytoma $($ Phaeo +$)$ and those without phaeochromocytoma (Phaeo-) is shown in table 2 . The previously described differences between the two groups have persisted. Missense mutations are significantly more frequent in the Phaeo + group $\left(\chi^{2}=15 \cdot 8, p<0.0005\right)$ and deletions, nonsense, and frameshift mutations are significantly more frequent in Phaeo - families $\left(\chi^{2}=10 \cdot 7, p<0.005\right)$.

To determine if there were age related differences in tumour risks with different classes of VHL gene mutations, we calculated cumulative age related risks for phaeochromocytoma, retinal and cerebellar haemangioblastomas, and RCC for the three most common types of germline mutations: large deletions, protein truncating mutations (nonsense and frameshift), and missense mutations. The age related risks for each tumour were similar in the deletion and protein truncating mutation groups (data not shown) and so these groups were combined and compared to missense mutations. The results of this analysis are shown in figs 1 to 4 . These clearly show that the cumulative risk of phaeochromocytoma is

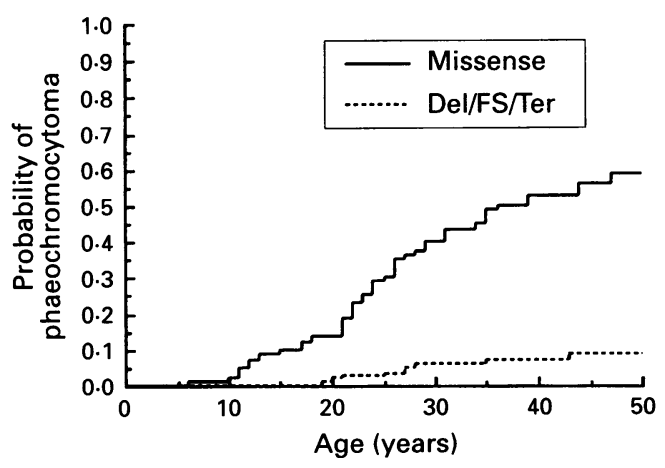

Figure 1 Age related risks of phaeochromocytoma in patients with missense VHL gene mutations and large deletions, frameshift deletions and insertions, and nonsense mutations (Del/FS/Ter).

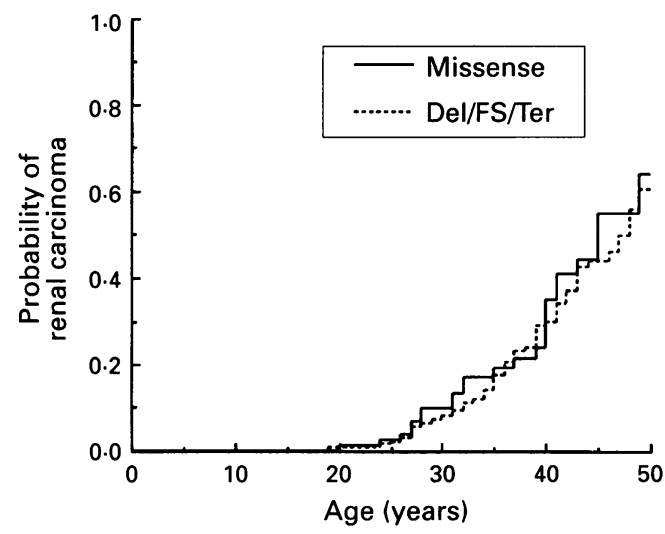

Figure 2 Age related risks of renal cell carcinoma in patients with missense VHL gene mutations and large deletions, frameshift deletions and insertions, and nonsense mutations (Del/FS/Ter).

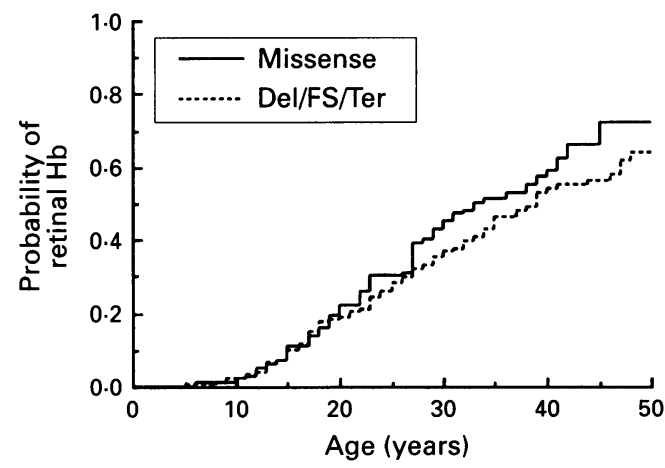

Figure 3 Age related risks of retinal haemangioblastoma in patients with missense VHL gene mutations and large deletions, frameshift deletions and insertions, and nonsense mutations (Del/FS/Ter).

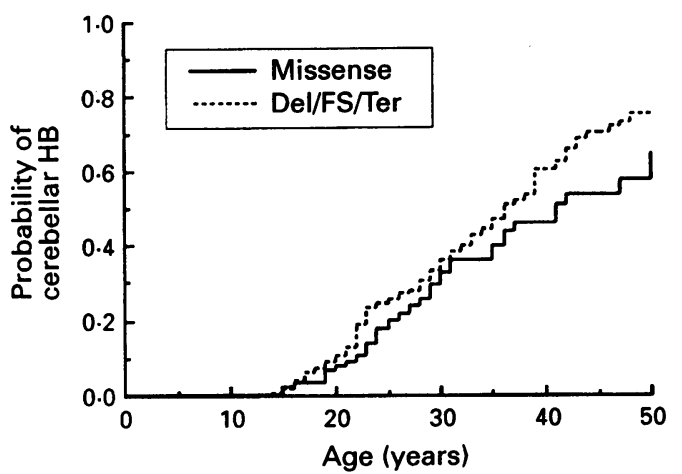

Figure 4 Age related risks of cerebellar

haemangioblastoma in patients with missense VHL gene mutations and large deletions, frameshift deletions and insertions, and nonsense mutations (Del/FS/Ter).

much higher in patients with missense mutations $(40 \%$ and $59 \%$ at ages 30 and 50 years respectively) than those with deletions/protein truncation mutations $(6 \%$ and $9 \%$ at 30 and 50 years respectively) (fig 1 ). Missense mutations are heterogeneous, but two common mutations (C712T and G713A) both cause missense mutations at codon 167 (previously codon 238 ${ }^{5910}$ ) (Arg167Trp and Arg167Gln, respectively) and are associated with a high risk of phaeochromocytoma: $53 \%$ and $82 \%$ at ages 30 and 50 years, compared to $20 \%$ and $25 \%$ at 30 and 50 years for non-codon 167 missense 
mutations. There was no major difference in the age related risk of renal cell carcinoma between the two groups of mutations (fig 2): deletion/protein truncation risks were $8 \%$ and $60 \%$ at ages 30 and 50 years, compared to $10 \%$ and $64 \%$ in the missense mutation group at the same ages. Age related risks for haemangioblastoma were similar in the two mutation groups, with the age related risks of cerebellar haemangioblastoma slightly less (35\% and $64 \%$ $v 38 \%$ and $75 \%$ at ages 30 and 50 years) and retinal haemangioblastoma slightly higher ( $45 \%$ and $72 \% v 37 \%$ and $64 \%$ at ages 30 and 50 years) in the missense mutation group than in the deletion/protein truncation group (figs 3 and 4 ). To determine if certain mutation types were associated with more severe disease and a higher mortality, actuarial survival curves were calculated for missense mutations and deletion/ protein truncating mutations. Median survival was 48 years in the deletion/protein truncation group and 49 years in the missense mutation group.

\section{Discussion}

Together with our previous reports, ${ }^{911}$ this paper describes the largest single centre collection of germline VHL mutations. Although the spectrum and distribution of mutations are similar to that found in studies from the USA and continental Europe ${ }^{1014}$ (B Zbar, personal communication), the incidence of large germline deletions detected by Southern analysis is significantly higher than that reported from Japan. ${ }^{15}$ As there is little evidence for founder effects for VHL gene deletions in the UK population, ${ }^{16}$ intragenic DNA sequences likely to give rise to deletions (for example, intronic repeats) may be less common in the Japanese population. Also, frequent intragenic mutations (for example, C694T, C712T, and G713A) do not show evidence of common haplotype and occur at $\mathrm{CpG}$ dinucleotides which are known to have a high mutability. ${ }^{13}$ Although Brauch et $a l^{17}$ have reported a founder effect for a VHL missense mutation (C505T) in 14 German and two USA families, this mutation is associated with a low risk of renal cell carcinoma (which is the leading cause of death in VHL disease) and therefore may have less effect on reproductive fitness than other VHL mutations. Recently, the translation initiation site within the VHL gene has been defined. ${ }^{12}$ This corresponds to codon 72 in original numbering of the $\mathrm{ORF}^{510}$ and the revised codon numbering used in this paper starts at the translation initiation site. Although the most $5^{\prime}$ portion of the open reading frame is now known not to be translated, it does contain VHL promoter activity. ${ }^{18}$ Nevertheless, we did not find mutations within this putative promoter region. The VHL gene product complexes with elongins $B$ and $C$ so preventing assembly of the elongin heterotrimer complex, which activates transcription elongation by RNA polymerase II. ${ }^{6-8}$ The elongin binding domain in the VHL protein is represented by codons 157-189 and many germline and so- matic VHL gene mutations are predicted to affect the integrity of this region. ${ }^{19}$

The detection of a germline VHL mutation allows presymptomatic diagnosis and improves the management of VHL families. Using conventional techniques (Southern, SSCP, and heteroduplex analyses), we achieved a mutation detection rate of $73 \%$. Patients without detectable mutations could have coding sequence changes not detected by SSCP or heteroduplex analysis, intermediate sized deletions removing whole exons that are too small to detect on Southern analysis, or mutations within regulatory sequences or introns, but the detection of coding sequence mutations by semiautomated DNA sequencing in eight subjects showed that SSCP and heteroduplex are not completely efficient in detecting intragenic mutations. The addition of VHL gene sequencing increased mutation detection efficiency from $73 \%$ to $81 \%$, despite the fact that the high GC content of exon 1 precluded sequencing of sufficient clarity to allow accurate mutation detection in a majority of samples.

It is clear that missense mutations are associated with a higher risk of phaeochromocytoma. Although missense mutations at codon 167 are associated with a particularly high risk of phaeochromocytoma, codon 167 mutation families cannot account for all the association between missense mutations and phaeochromocytoma and other mutations contribute to this effect. The identification of a VHL mutation can be used not only to provide accurate presymptomatic diagnosis within the family, but also to predict the likely phenotype. VHL kindreds with missense mutations (especially codon 167 mutations and other missense mutations reported in phaeochromocytoma positive families) require careful surveillance for phaeochromocytoma as this is frequently the first manifestation. Patients with other types of mutations will generally be at lower risk of developing phaeochromocytoma, but should continue to be monitored by annual urinary VMAs and metadrenaline estimations as a minority will develop this complication. The tumour specific age related risks we have calculated provide valuable data for counselling VHL families. In addition, they may also provide clues to the possible molecular mechanisms for the genotype-phenotype correlation. For example, the low risk of phaeochromocytoma in families with deletions and protein truncating mutations is not because these mutations cause more severe disease so that patients do not survive to develop phaeochromocytoma as (1) the age related risk of phaeochromocytoma was unequivocally higher for missense mutations and (2) deletion and protein truncating mutations were not associated with reduced survival. A further possibility would be that missense mutations might act in a dominant negative mode causing more severe disease, with an earlier onset for all tumours including phaeochromocytoma. Missense mutations in the p53 tumour suppressor gene may function in this manner and, in transgenic mouse models, are associated with higher age related tumour risks than p53 knockout 
mutations. ${ }^{20}$ If similar "dominant negative" effects were operative in VHL disease it would be expected that, in addition to a higher risk of phaeochromocytoma, missense mutations would be associated with an increased age related risk for haemangioblastomas and renal cell carcinomas and there was no evidence of this. Our results would be more compatible with phaeochromocytoma associated missense mutations having tissue specific effects, possibly because the VHL protein has several functions, the importance of which varies from tissue to tissue, or because the proteins which interact with VHL differ in different tissues. In support of this hypothesis are the results of other genotype-phenotype correlations. The Black Forest mutation reported by Brauch et $a l^{17}$ is associated with retinal and cerebellar haemangioblastoma and phaeochromocytoma, but a low risk of renal cell carcinoma. In addition, we have detected germline VHL gene mutations in families with familial phaeochromocytoma without other evidence of VHL disease suggesting that some missense VHL gene mutations may only predispose to phaeochromocytoma. ${ }^{2122}$ The investigation of the relationship between VHL mutations and the elongin binding function (and other putative VHL functions) of the VHL protein may provide a basis for elucidating the molecular mechanisms responsible for these intriguing genotype-phenotype correlations.

We thank the Guide Dogs for the Blind Association, Action Research, and the Cancer Research Campaign (CRC) for financial support. We are grateful to the many colleagues who have provided us with access to families and to the patients and have provided us with access to families
relatives who took part in this study.

1 Maher ER, Iselius L, Yates JRW, et al. Von Hippel-Lindau disease: a genetic study. $\mathcal{F}$ Med Genet 1991;28:443-7.

2 Maher ER, Yates JR, Harries R, et al. Clinical features and natural history of von Hippel-Lindau disease. $Q \mathcal{F} \mathrm{Med}$ 1990;77:1151-63.

3 Maher ER. Von Hippel-Lindau disease. Eur $\mathcal{F}$ Cancer 1994; 30:1987-90.

4 Seizinger BR, Rouleau GA, Ozelius LJ, et al. Von HippelLindau disease maps to the region of chromosome 3
a associated with renal cell carcinoma. Nature 1988;332: 268-9.

5 Latif F, Tory K, Gnarra J, et al. Identification of the von Hippel-Lindau disease tumour suppressor gene. Science 1'993;260:1317-20.

6 Duan DR, Pause A, Burgess WH, et al. Inhibition of transcription elongation by the VHL tumor suppressor protein. Science 1995;269:1402-6.

7 Aso T, Lane WS, Conaway JW, Conaway RC. Elongin (SIII): a multisubunit regulator of elongation by RNA polymerase II. Science 1995;269:1439-43.

8 Kibel A, Iliopoulos O, DeCaprio JA, Kaelin WG. Binding of the von Hippel-Lindau tumor suppressor protein to of the von Hippel-Lindau tumor suppresso
elongin B and C. Science 1995;269:1444-6.

9 Crossey PA, Richards RM, Foster K, et al. Identification of intragenic mutations in the von Hippel-Lindau disease tumour suppressor gene and correlation with disease phenotype. Hum Molec Genet 1994;3:1303-8.

10 Chen F, Kishida T, Yao M, et al. Germ line mutations in the von Hippel-Lindau disease tumor suppressor gene: correlations with phenotype. Hum Mutat 1995;5:66-75.

11 Richards FM, Crossey PA, Phipps ME, et al. Detailed mapping of germline deletions of the von Hippel-Lindau disease tumour suppressor gene. Hum Molec Genet 1994; 3:595-8.

12 Iliopoulos O, Kibel A, Gray S, Kaelin WG. Tumor suppression by the human von Hippel-Lindau gene product. pression by the human von $1995 ; 1: 822-6$.

13 Cooper DN, Youssoufian $\mathrm{H}$. The $\mathrm{CpG}$ dinucleotide and human genetic disease. Hum Genet 1988;78:151-5.

14 Whaley JS, Naglich J, Gelbert L, et al. Germ-line mutations in the von Hippel-Lindau tumor-suppressor gene are similar to somatic von Hippel-Lindau aberrations in sporadic renal cell carcinoma. Am f Hum Genet 1994;55:1092-102.

15 Clinical research group for VHL disease in Japan. Germline mutations in the von Hippel-Lindau disease (VHL) gene in Japanese VHL. Hum Molec Genet 1995;4:2233-7.

16 Richards FM, Payne SJ, Zbar B, Affara NA, FergusonSmith MA, Maher ER. Molecular analysis of de novo germline mutations in the von Hippel-Lindau disease gene. Hum Molec Genet 1995;4:2139-43.

17 Brauch $\mathrm{H}$, Kishida $\mathrm{T}$, Glavac D, et al. Von Hippel Lindau disease with pheochromocytoma in the Black Forest region of Germany: evidence for a founder effect. Hum Genet 1995;95:551-6.

18 Kuzmin I, Duh FM, Latif F, Geil L, Zbar B, Lerman MI Identification of the promotor of the human von Hippel Identification of the promotor of the human von Hippel
Lindau disease tumor suppressor gene. Oncogene 1995; 10:2185-94

19 Kishida T, Stackhouse T, Chen F, Lerman MI, Zbar B. Cellular proteins that bind the von Hippel-Lindau disease gene product: mapping of binding domains and the effect of missense mutations. Cancer Res 1995;55:4544-8.

20 Vogel H, Morris D, Bradley A, Bernstein A, Donehoer LA. A mutant p53 transgene accelerates tumour development in heterozygous but not nullizygous p53-deficient mice. Nature Genet 1995;5:305-11.

21 Crossey PA, Eng C, Ginalska-Malinowska M, et al. Molecular genetic diagnosis of von Hippel-Lindau disease in familial phaeochromocytoma. $\mathcal{F}$ Med Genet 1995;32: 885-6.

22 Neumann HPH, Eng C, Mulligan L, et al. Consequences of direct genetic testing for germline mutations in the clinical management of families with multiple endocrine neoplasia type 2. $\mathscr{f} A M A 1995 ; 274: 1149-51$. 